\title{
A GENERALIZATION OF SPERNER'S THEOREM
}

\author{
D. E. DAYKIN, P. FRANKL, C. GREENE and A. J. W. HILTON
}

(Received 1 August 1980)

Communicated by W. D. Wallis

\begin{abstract}
Some generalizations of Sperner's theorem and of the LYM inequality are given to the case when $A_{1}, \ldots, A_{t}$ are $t$ families of subsets of $\{1, \ldots, m\}$ such that a set in one family does not properly contain a set in another.

1980 Mathematics subject classification (Amer. Math. Soc.): 05 A 20.

In this note we generalize Sperner's theorem [3] that "a Sperner family (or clutter, or antichain) of subsets of the finite set $\{1, \ldots, m\}$ contains at most $\left(\begin{array}{c}m \\ m / 2\end{array}\right)$ sets", to the case where $\mathbb{Q}_{1}, \ldots, \mathbb{Q}_{t}$ are $t$ families of subsets of $\{1, \ldots, m\}$ such that a set in one family does not properly contain a set in another. We also generalize the LYM inequality to the case and give another interesting inequality.
\end{abstract}

THEOREM. Let $t \geqslant 2, m \geqslant 2$. Let $\mathbb{Q}_{1}, \ldots, \mathbb{Q}_{t}$ be $t$ sets of subsets of $\{1, \ldots, m\}$ such that

$$
A_{i} \in \mathbb{Q}_{i}, A_{j} \in \mathbb{Q}_{j}, i \neq j \Rightarrow A_{i} \text { does not properly contain } A_{j} .
$$

Let $\beta_{i j}$ be the number of sets of cardinality $i$ in $\mathbb{Q}_{j}$ and let $\gamma_{i}=\beta_{i 1}+\cdots+\beta_{i t}$. Then

(i) $\sum_{i=0}^{m} \gamma_{i} /\left(\begin{array}{c}m \\ i\end{array}\right)<\max (t, m+1)$,

(ii) $\left|\mathbb{Q}_{1}\right|+\cdots+\left|\mathbb{Q}_{t}\right| \leqslant \max \left(2^{m}, t\left({ }_{(m / 2)}^{m}\right)\right)$,

(iii) $\left|Q_{1}\right|+\cdots+\left|Q_{t}\right| \leqslant 2^{m}+s t-2^{m}\left(\begin{array}{c}m \\ m / 2\end{array}\right)^{-1} s$, where $s$ is the number of subsets of $\{1, \ldots, m\}$ which occur in more than one $\mathbb{Q}_{i}$. 
Remarks. 1. All the bounds are best possible. If $t \geqslant m+1$ then we can take each $\mathcal{Q}_{i}$ to consist of all subsets of some given size $i$. If $m+1>t$ we can have $\mathbb{Q}_{1}$ consisting of all subsets of $\{1, \ldots, m\}$, and $\left|\mathscr{Q}_{2}\right|=\cdots=\left|\mathbb{Q}_{t}\right|=0$. Then $\gamma_{i}=\left(\begin{array}{c}m \\ i\end{array}\right)$.

2. (ii) follows from (iii) since, by Sperner's theorem

$$
s \leqslant\left(\left[\begin{array}{c}
m \\
2
\end{array}\right]\right)
$$

However, we give another derivation of (ii) as well.

Proof of (i). Let

$$
x=\sum_{i=0}^{m} \gamma_{i} \frac{m !}{\left(\begin{array}{c}
m \\
i
\end{array}\right)} .
$$

Then

$$
x=\sum_{j=1}^{t} \sum_{a \in \mathbb{Q}_{j}}(\text { the number of maximal chains through } a),
$$

since the number of maximal chains through a set $a$ of cardinality $i$ is $i(i-1) \cdots 1 .(m-i)(m-i-1) \cdots 1=m ! /\left(\begin{array}{c}m \\ i\end{array}\right)$. The number of maximal chains is $m$ !. Therefore

$x \leqslant m$ ! (the number of times a maximal chain can be counted in (1))

$$
\begin{aligned}
& =m !\left\{\begin{array}{l}
t \text { if the maximal chain meets only one } a \in \mathbb{Q}_{1} \cup \cdots \cup \mathbb{Q}_{t}, \\
m+1 \text { if the maximal chain meets more than one } \\
a \in \mathbb{Q}_{1} \cup \cdots \cup \mathbb{Q}_{t}, \text { for then each such } a \text { must be in the same } \mathbb{Q}_{i} .
\end{array}\right. \\
& \leqslant m ! \max (t, m+1) .
\end{aligned}
$$

(i) now follows by dividing by $m$ !.

Proof of (ii). We use the following result of Kleitman and Greene [2] (which we have specialized for our purpose). Let $\lambda$ be a real valued function defined on the subsets of $\{1, \ldots, m\}$. Let $C$ be the set of all maximal chains. If $B$ is any set of subsets of $\{1, \ldots, m\}$, then

$$
\sum_{b \in B} \frac{\lambda_{b}}{\left(\begin{array}{c}
m \\
|b|
\end{array}\right)} \leqslant \max _{C \in \mathcal{C}} \sum_{b \in C \cap B} \lambda_{b} .
$$

To apply this result, let $\lambda_{b}=\left(\begin{array}{c}m \\ |b|\end{array}\right) \times\left(\right.$ the number of $\mathcal{Q}_{i}$ 's containing $b$ ). Then

$$
\left|\mathbb{Q}_{1}\right|+\cdots+\left|\mathbb{Q}_{t}\right|=\sum_{a \in \cup \mathbb{Q}_{i}} \frac{\lambda_{a}}{\left(\begin{array}{c}
m \\
|a|
\end{array}\right)} \leqslant \max _{C \in \mathcal{C}} \sum_{a \in C \cap\left(\cup \mathbb{Q}_{i}\right)} \lambda_{a} .
$$


If one of the $a$ 's in the chain $C$ occurs in more than one $\mathbb{Q}_{i}$ then $\lambda_{a} \leqslant t$ and there is only one element in $C \cap\left(\cup Q_{i}\right)$ so we get

$$
\left|\mathbb{Q}_{1}\right|+\cdots+\left|\mathbb{Q}_{t}\right| \leqslant t \max _{a \in \mathbb{Q}_{1} \cup \cdots \cup \mathbb{Q}_{t}}\left(\begin{array}{c}
m \\
|a|
\end{array}\right) \leqslant t\left(\left[\begin{array}{c}
m \\
\left.\frac{m}{2}\right]
\end{array}\right]\right) .
$$

If each $a$ in $C$ occurs in at most one $\mathscr{C}_{i}$ then

$$
\left|\mathscr{Q}_{1}\right|+\cdots+\left|\mathbb{Q}_{i}\right| \leqslant \sum_{i=0}^{m}\left(\begin{array}{c}
m \\
i
\end{array}\right)=2^{m}
$$

This proves (ii).

Proof of (iii). To prove (iii), first we prove the following lemma which is of some interest in its own right.

Lemma. Let $T$ be a family of subsets of $\{1, \ldots, m\}$. Then the probability that a given set is in $T$ is not greater than the probability that the given set is in a maximal chain which meets $T$. ("meets" here means that the maximal chain contains a member of $T$.)

Proof of THE Lemma. For $0 \leqslant i \leqslant m$, let $t_{i}$ be the number of sets of $T$ of cardinality $i$. Let

$$
\mu=\max _{0<i<m} \frac{t_{i}}{\left(\begin{array}{c}
m \\
i
\end{array}\right)}=\frac{t_{i_{0}}}{\left(\begin{array}{c}
m \\
i_{0}
\end{array}\right)} .
$$

Then for $0 \leqslant i \leqslant m, t_{i} \leqslant \mu\left(\begin{array}{c}m \\ i\end{array}\right)$ so $\sum_{i=0}^{m} t_{i} \leqslant \mu \sum_{i=0}^{m}\left(\begin{array}{c}m \\ i\end{array}\right)=\mu 2^{m}$. Therefore

$$
\begin{aligned}
\frac{|T|}{2^{m}} & =\frac{\sum_{i=0}^{m} t_{i}}{2^{m}} \leqslant \mu=\frac{t_{i_{0}}}{\left(\begin{array}{c}
m \\
i_{0}
\end{array}\right)}=\frac{t_{i_{0}}}{m !}\left(i_{0}\right) !\left(m-i_{0}\right) ! \\
& =\frac{1}{m !}\left(\begin{array}{c}
\text { the number of maximal chains which meet } \\
\text { a member of } T \text { of size } i_{0} .
\end{array}\right) \\
& \leqslant \frac{\text { the number of maximal chains which meet } T}{\text { the total number of maximal chains }} .
\end{aligned}
$$

The lemma now follows.

Now to return to the proof of (iii). Let $S=\{a: a$ lies in more than one of $\left.\mathbb{Q}_{1}, \ldots, \mathbb{Q}_{t}\right\}$ and let $T=\left\{a: a \in\left(\mathbb{Q}_{1} \cup \cdots \cup \mathbb{Q}_{t}\right) \backslash S\right\}$. Then $S$ is a Sperner family, $|S|=s$ and $S$ and $T$ are incomparable. 
The probability that a set of size $i$ is in a given chain is

$$
\frac{1}{\left(\begin{array}{c}
m \\
i
\end{array}\right)}>\frac{1}{\left(\left[\begin{array}{c}
m \\
\left.\frac{m}{2}\right]
\end{array}\right]\right)}
$$

Therefore, the probability that a given chain meets $S$ is at least

$$
\sum_{a \in S} \frac{1}{\left(\begin{array}{c}
m \\
{\left[\frac{m}{2}\right]}
\end{array}\right]}=\frac{s}{\left(\left[\begin{array}{c}
m \\
\left.\frac{m}{2}\right]
\end{array}\right]\right.}
$$

Since $S$ and $T$ are incomparable, it follows from the lemma that the probability that a given maximal chain meets $S$ or $T$ is at least

$$
\frac{|S|}{\left(\left[\begin{array}{l}
m \\
\left.\frac{m}{2}\right]
\end{array}\right]\right.}+\frac{|T|}{2^{m}}
$$

and so it follows that

$$
\frac{|S|}{\left(\left[\frac{m}{2}\right]\right)}+\frac{|T|}{2^{m}}<1
$$

and therefore

$$
|T| \leqslant 2^{m}-\frac{2^{m} s}{\left(\left[\begin{array}{c}
m \\
\frac{m}{2}
\end{array}\right]\right)}
$$

Now we have that

$$
\left|\mathfrak{Q}_{1}\right|+\cdots+\left|\mathbb{Q}_{t}\right| \leqslant|T|+t|S| \leqslant 2^{m}+s t-\frac{2^{m}}{\left(\left[\begin{array}{c}
m \\
2
\end{array}\right]\right)}
$$

\section{References}

[1] C. Greene and D. J. Kleitman, 'Proof techniques in the theory of finite sets', Studies in Combinatorics, edited by G.-C. Rota (MAA Studies in Mathematics 17 (1978), 22-79).

[2] D. J. Kleitman, 'On an extremal property of antichains in partial orders. The LYM property and some of its implications and applications', Combinatorics edited by M. Hall and J. H. van Lint, (Math. Centre Tracts 55, Amsterdam, 1974, 77-90).

[3] E. Sperner, 'Ein Satz über Untermengen einer endlichen Menge', Math. Z. 27 (1928), 544-548. 
Department of Mathematics The University of Reading Reading, RG6 2AX

United Kingdom

Department of Mathematics Haverford College Haverford, Pennsylvania 19041 U.S.A.
Centre national de la recherche scientifique 515 quai Anatole France 75700 Paris France Department of Mathematics The University of Reading Reading, RG6 2AX United Kingdom 